\title{
Test of Time-Reversal Symmetry in the Proton-Deuteron Scattering
}

\author{
Yu. N. Uzikov \\ Joint Institute for Nuclear Researches, Joliott-Curie 6, Dubna, 141980, Russia \\ Physics Department, Moscow State University, Moscow, 119991 Russia \\ uzikov@jinr.ru \\ A. A. Temerbayev \\ L.N. Gumilyov Euroasian National University, K.Munaitpasov 5, Astana 010000, Kazakhstan \\ adastra.77@mail.ru
}

Published 29 February 2016

\begin{abstract}
The integrated cross section $\widetilde{\sigma}$ for a special type of double polarized proton-deuteron scattering constitutes a null test for time-invariance violating but P-parity conserving effects. Using Glauber theory for the $p d$ elastic scattering and different types of phenomenological T-odd P-even NN-interactions we show that the contribution of the lowest mass meson exchange, i.e. the $\rho$-meson, to the null-test signal $\widetilde{\sigma}$ vanishes. Variation of the cross section $\widetilde{\sigma}$ due to strong hadronic and Coulomb interaction is studied and its energy dependence is calculated in the $\mathrm{GeV}$ region.
\end{abstract}

Keywords: Time-invariance; proton-deuteron scattering; double-polarization.

PACS numbers: 24.80.+y, 25.10.+s, 11.30.Er, 13.75.Cs

\section{Introduction}

Time-invariance-violating (T-odd) P-parity conserving (P-even) (TVPC) interactions do not arise on the fundamental level within the standard model. This type of interaction can be generated by radiative corrections to the T-odd $\mathrm{P}$-odd interaction discovered in physics of kaons and B-mesons. However in such a case its intensity is too small to be observed in experiments at present. ${ }^{1}$ Thus, observation of TVPC effects would be considered as indication to physics beyond the standard model.

As was shown in Ref. 2, the total polarized cross section $\widetilde{\sigma}$ of the proton-deuteron scattering with vector polarization of the proton $p_{y}^{p}$ and tensor polarization of the deuteron $P_{x z}$ constitutes a null-test of TVPC effects. The dedicated experiment is planned at $\mathrm{COSY}^{3}$ at proton beam energy $135 \mathrm{MeV}$ which is motivated by theoretical estimations of the TVPC effects. ${ }^{4}$ Analysis of the TVPC null-test signal ${ }^{4}$ was

This is an Open Access article published by World Scientific Publishing Company. It is distributed under the terms of the Creative Commons Attribution 3.0 (CC-BY) License. Further distribution of this work is permitted, provided the original work is properly cited. 
done within the nonmesonic deuteron breakup channel $p d \rightarrow p p n$ estimated in the impulse (single scattering) approximation. Here we use the spin-dependent formalism $^{5}$ of the Glauber theory to calculate the cross section $\widetilde{\sigma}$. The formalism includes full spin dependence of elementary $\mathrm{pN}$-amplitudes and $\mathrm{S}$ - and D-components of the deuteron wave function. This formalism was used ${ }^{6}$ to calculate non-polarized differential cross section and spin observables of the elastic $p d$ scattering and total polarized $p d$ cross sections at energy of the TRIC experiment $135 \mathrm{MeV}$. We further develop the formalism to account for the Coulomb and TVPC interactions.

\section{Elements of formalism}

The total cross section of the $p d$ scattering has the form ${ }^{6}$

$$
\sigma_{t o t}=\sigma_{0}+\sigma_{1} \mathbf{p}^{p} \cdot \mathbf{p}^{d}+\sigma_{2}\left(\mathbf{p}^{p} \cdot \hat{\mathbf{k}}\right)\left(\mathbf{p}^{d} \cdot \hat{\mathbf{k}}\right)+\sigma_{3} P_{z z}+\widetilde{\sigma} p_{y}^{p} P_{x z}^{d}
$$

where $\mathbf{p}^{p}\left(\mathbf{p}^{d}\right)$ is the vector polarization of the initial proton (deuteron) and $P_{z z}$ and $P_{x z}$ are the tensor polarizations of the deuteron. The $\mathrm{OZ}$ axis is directed along the proton beam momentum $\hat{\mathbf{k}}, \mathrm{OY} \uparrow \uparrow \mathbf{p}^{p}, \mathrm{OX} \uparrow \uparrow\left[\mathbf{p}^{p} \times \hat{\mathbf{k}}\right]$. In Eq. (1) the terms $\sigma_{i}$ with $i=0,1,2,3$ are non-zero only for $\mathrm{T}$-even P-even interaction and the last term $\widetilde{\sigma}$ constitutes a null-test signal of T-invariance violation with $\mathrm{P}$-parity conservation. Hadronic amplitudes of $p N$ scattering are taken $\mathrm{as}^{5}$

$$
\begin{array}{r}
M_{N}\left(\mathbf{p}, \mathbf{q} ; \boldsymbol{\sigma}, \boldsymbol{\sigma}_{N}\right)=A_{N}+C_{N} \boldsymbol{\sigma} \hat{\mathbf{n}}+C_{N}^{\prime} \boldsymbol{\sigma}_{N} \hat{\mathbf{n}}+B_{N}(\boldsymbol{\sigma} \hat{\mathbf{k}})\left(\boldsymbol{\sigma}_{N} \hat{\mathbf{k}}\right)+ \\
+\left(G_{N}+H_{N}\right)(\boldsymbol{\sigma} \hat{\mathbf{q}})\left(\boldsymbol{\sigma}_{N} \hat{\mathbf{q}}\right)+\left(G_{N}-H_{N}\right)(\boldsymbol{\sigma} \hat{\mathbf{n}})\left(\boldsymbol{\sigma}_{N} \hat{\mathbf{n}}\right)
\end{array}
$$

where orts $\hat{\mathbf{q}}, \hat{\mathbf{k}}$ and $\hat{\mathbf{n}}$ are defined as unit vectors along the vectors $\mathbf{q}=\mathbf{p}-\mathbf{p}^{\prime}, \mathbf{k}=$ $\mathbf{p}+\mathbf{p}^{\prime}$ and $\mathbf{n}=[\mathbf{k} \times \mathbf{q}]$, respectively; $\mathbf{p}\left(\mathbf{p}^{\prime}\right)$ is the initial (final) proton momentum. We consider the following terms of the TVPC NN interaction which were under discussion in Ref. 4:

$$
\begin{array}{r}
t_{p N}=h_{N}\left[(\boldsymbol{\sigma} \cdot \mathbf{k})\left(\boldsymbol{\sigma}_{N} \cdot \mathbf{q}\right)+\left(\boldsymbol{\sigma}_{N} \cdot \mathbf{k}\right)(\boldsymbol{\sigma} \cdot \mathbf{q})-\frac{2}{3}\left(\boldsymbol{\sigma}_{N} \cdot \boldsymbol{\sigma}\right)(\mathbf{k} \cdot \mathbf{q})\right] / m_{p}^{2}+ \\
\quad+g_{N}\left[\boldsymbol{\sigma} \times \boldsymbol{\sigma}_{N}\right] \cdot[\mathbf{q} \times \mathbf{k}] / m_{p}^{2}+g_{N}^{\prime}\left(\boldsymbol{\sigma}-\boldsymbol{\sigma}_{N}\right) \cdot i[\mathbf{q} \times \mathbf{k}]\left[\boldsymbol{\tau} \times \boldsymbol{\tau}_{N}\right]_{z} / m_{p}^{2}
\end{array}
$$

Here $\boldsymbol{\sigma}\left(\boldsymbol{\sigma}_{N}\right)$ is the Pauli matrix acting on the spin state of the proton (nucleon $N=p, n), \boldsymbol{\tau}\left(\boldsymbol{\tau}_{N}\right)$ is the corresponding matrix acting on the isospin state; $m_{p}$ is the proton mass. In the framework of the phenomenological meson exchange interaction the term $g^{\prime}$ corresponds to $\rho$-meson exchange, and $h$-term provides the axial meson $h_{1}$ exchange with $h_{N}=-i 2 G_{h} \bar{G}_{h} F\left(q^{2}\right)\left(m_{h}^{2}+\mathbf{q}^{2}\right)^{-1}$, where $G_{h}\left(\bar{G}_{h}\right)$ is the ordinary (TVPC) $h N N$ coupling constant, $m_{h}$ is the h-meson mass and $F\left(q^{2}\right)$ is the $h N N$ vertex form factor. ${ }^{4}$

Using the generalized optical theorem we find $\widetilde{\sigma}=-4 \sqrt{\pi} \operatorname{Im} \frac{2}{3} \widetilde{g}$. For single scattering mechanism the amplitude $\widetilde{g}$ vanishes within the Glauber theory. For double scattering mechanism with $p N$-amplitudes (2) and (3) within the S-wave approxi- 
mation for the deuteron wave function we find

$$
\widetilde{g}=\frac{i}{4 \pi m_{p}} \int_{0}^{\infty} d q q^{2} S_{0}^{(0)}(q)\left[C_{n}^{\prime}(q)\left(g_{p}-h_{p}\right)+C_{p}^{\prime}(q)\left(g_{n}-h_{n}\right)\right],
$$

where $S_{0}^{(0)}$ is the S-wave elastic form factor of the deuteron in notations of Ref. 5 .

\section{Numerical results and discussion}

The results of our calculations ${ }^{6}$ for non-polarized differential cross section, vector $A_{y}$ and tensor $A_{i j}$ analyzing powers, spin correlations parameters $C_{i, j}, C_{i j, k}$ are in reasonable agreement with the available experimental data at $135 \mathrm{MeV}$ and $250 \mathrm{MeV}$ in forward hemisphere. We found also that account for Coulomb effects improves agreement with the data at these energies at small angles $\theta_{c m} \leq 20^{\circ}-30^{\circ}$. The obtained results ${ }^{6}$ allow one to conclude that the Glauber theory is quite suitable for study of the null-test signal for TVPC effects in the $p d$-scattering because this signal is not imitated by the strong background caused by T-even P-even interactions. Our approach is more general than the approximation used in previous study. ${ }^{4}$

The $g^{\prime}$-term gives zero contribution to $\widetilde{g}$ in Eq. (4). Account for the D-wave does not change this result. Therefore, the $\rho$-meson exchange in general allowed as the lowest mass meson in the TVPC $N N$ interaction $^{7}$ and expected to dominate the TVPC $N N$ interaction, does not contribute to the null-test signal $\widetilde{\sigma}$. Contribution of other more heavy mesons is expected to be less important due to $N N$ repulsive core at short distances between nucleons. A microscopic T-violating optical potential for nucleon-nucleus interaction was derived in Ref. 8 starting from the T-violating $\rho$-meson interaction between nucleons and the corresponding coupling constant of the $\rho$-meson to the nucleon $\bar{g}_{\rho}$ is widely used ${ }^{7,9}$ as a measure of intensity of the TVPC effects. One can see, however, for the $p d$ scattering this parameter can not be applied directly as a scale of the TVPC interactions.

Now we consider a role of Coulomb effects in the cross section $\widetilde{\sigma}$. The Coulomb $p p$ scattering being T-even P-even interaction cannot generate the TVPC amplitude $\widetilde{g}$ within the single scattering mechanism, therefore its contribution to $\widetilde{g}$ is zero in this approximation. To account for the Coulomb interaction within the double scattering mechanism one should add the $p p$-scattering amplitude $f_{p p}^{C}$ to the pure hadronic T-even P-even $p p$ amplitude $M_{p}$ in Eq.(2). The amplitude $f_{p p}^{C}$ does not depend on spins and therefore is added only to the spin-independent term $A_{p}$. However, the $A_{p}$ term is excluded from the TVPC amplitude (4) due to its specific spin structure. The $C_{p}^{\prime}$ is the only factor in Eq.(4) which contains Coulomb effects via the $A_{p}$ because of the relation ${ }^{5,10} C_{p}^{\prime}=C_{p}+i\left(q / 2 m_{p}\right) A_{p}$. When substituting this amplitude into Eq. (4) and making integration over $q$, we find that the singularity of the Coulomb amplitude $f_{p p}^{C}\left(\theta_{p p}\right)$ at $\theta_{p p}=0$ does not lead to divergence of the null-test amplitude $\widetilde{g}$. Energy dependence of $\widetilde{\sigma}$ calculated for the $h$ term with $G_{h}^{2} / 4 \pi=1.56, m_{h}=1.17$ $\mathrm{GeV}$ in units of the unknown $h N N$ coupling constant $\phi_{h}=\bar{G}_{h} / G_{h}$ is shown in Fig.1. The $C_{p}$ and $A_{p}$ amplitudes are taken from Ref.11. The $g$-term leads to a 


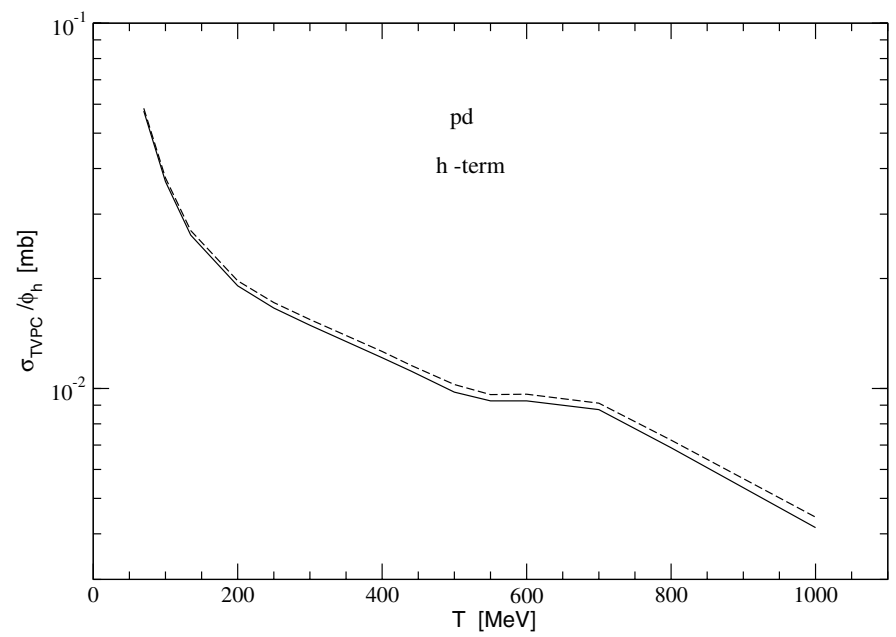

Fig. 1. The calculated energy dependence of the TVPC cross section $|\widetilde{\sigma}|$ for the $h$-term in units of the constant $\phi_{h}=\bar{G}_{h} / G_{h}$ with the Coulomb interaction included (dashed line) and excluded (full).

very similar energy dependence. One can see that energies $\sim 100 \mathrm{MeV}$ are more preferable than $\sim 1 \mathrm{GeV}$ to search for the TVPC effects.

\section{Summary}

The Glauber theory provides a theoretical basis for estimation of the TVPC effects in the double polarized $p d$ scattering at the energy of the experiment ${ }^{3}$ planned at COSY. Using this theory we find that the $\rho$-meson exchange between nucleons is vanishing in the TVPC signal $\widetilde{\sigma}$ in the $p d$ scattering whereas the $h$ and $g$ terms give non-zero contributions. We show also that the Coulomb interaction makes a minor influence on this observable.

\section{References}

1. I. B. Khriplovich, Nucl. Phys. B 352, 385 (1991).

2. H. E. Conzett, Phys. Rev. C 48, 423 (1993).

3. COSY Proposal N 215, "Test of Time reversal invariance in proton-deuteron scattering at COSY", Spokespersons: P. D. Eversheim, B. Lorentz, Yu. Valdau; http://donald.cc.kfa-juelich.de/wochenplan/List of all COSY-Proposals.shtml

4. M. Beyer, Nucl. Phys. A 560, 895 (1993).

5. M. N. Platonova, V. I. Kukulin. Phys. Rev. C 81, 014004 (2010).

6. A. A. Temerbayev, Yu. N. Uzikov, Yad. Fiz. 78, 38 (2015).

7. M. Simonius, Phys. Rev. Lett. 78, 4161 (1997).

8. J. Engel, C. R. Gould, V. Hnizdo, Phys. Rev. Lett. 73, 3508 (1994).

9. P. R. Huffman et al., Phys. Rev. C 55, 2684 (1997).

10. C. Sorensen, Phys. Rev. D 19, 1444 (1979).

11. R. A. Arndt, W. J. Briscoe, I. I. Strakovsky, R. I. Workman, Phys. Rev. C 76, 025209 (2007); http://gwdac.phys.gwu.edu. 